\title{
Investigation of laser-irradiated Ar cluster dynamics from K-shell x-ray emission measurements
}

\author{
F. Dorchies* and T. Caillaud, F. Blasco, C. Bonté, H. Jouin, S. Micheau, B. Pons, J. Stevefelt \\ CELIA, UMR 5107, CNRS - CEA - Université Bordeaux I, 33405 Talence, France
}

(Dated: April 26, 2005)

\begin{abstract}
Intense (up to a few $10^{17} \mathrm{~W} / \mathrm{cm}^{2}$ ) femtosecond (down to $40 \mathrm{fs}$ ) laser pulses are focused onto a partially clusterized Argon gas jet. The target was previously characterized and optimized in order to get a homogeneous and dense jet of clusters with a well controlled size. The interaction leads to x-ray emission that is absolutely calibrated and spectrally resolved using a high resolution timeintegrated spectrometer in the K-shell range (from 2.9 to $4.3 \mathrm{keV}$ ). X-ray spectra are investigated as a function of different laser temporal parameters such as the nanosecond pre-pulse contrast, the laser pulse duration, and the femtosecond delay between two different laser pulses. The cluster size ranges from 180 to $350 \AA$ and irradiation by laser pulses with both linear and circular polarization is investigated. The experimental results are discussed in terms of the laser-cluster interaction dynamics. They are compared with the predictions of collision-dominated nano-plasma models. However, further interaction processes are required in order to explain the observed characteristic lines demonstrating highly charged ions up to $\mathrm{Ar}^{16+}$.
\end{abstract}

PACS numbers: 52.50.Jm, 52.38.Ph, 32.30.Rj

\section{INTRODUCTION}

In the last decade, the irradiation of rare gas clusters with intense femtosecond lasers $\left(>10^{15} \mathrm{~W} / \mathrm{cm}^{2},<1\right.$ ps) has been extensively studied [1]. An anomalous x-uv and x-ray emission has been observed from the laser interaction with a high pressure rare gas jet and has been demonstrated to be correlated with a cluster production in the jet [2]. This result has been confirmed by other groups together with the detection of high energy electrons and highly charged state ions from this new type of laser target [3-7]. Using deuterium clusters, neutrons have also been produced via nuclear fusion reactions with a relatively small laser system [8]. Such a target is obtained by the rapid expansion of a high pressure rare gas through a nozzle. Its partial condensation into clusters leads to an intermediate media between gas (low average density) and solid (high local density) combining advantages of both targets : propagation through a large interaction volume and highly efficient absorption of laser energy (up to $95 \%$ [9]). Applications of the laser-cluster interaction have been proposed relying on these unique properties. In particular, high repetition rate laser-based radiation sources are expected without debris : in the $\mathrm{x}$-uv range for lithographic applications [10] and in the multi-keV range for ultra-fast $x$-ray science applications [11].

Its possible application to the ultrafast $\mathrm{x}$-ray science relies on the ability to produce x-ray bursts in the ps or sub-ps range. To our knowledge, only two experiments have been performed so far in order to determine the x-ray duration in the multi-keV range. The first one reported a few ns duration for the K-shell x-ray emis-

*Electronic address: dorchies@celia.u-bordeaux1.fr sion of Argon clusters ( $\sim 3 \mathrm{keV})$ [12]. This result has not been confirmed by the second group, since they have measured a duration of 100 ps for the M-shell of Xenon clusters $(\sim 1 \mathrm{keV})$, limited by their temporal resolution [13]. The determination of the duration of x-ray emission from clusters is still a great experimental and theoretical challenge. Its determination depends a lot on the model considered to describe the dynamics of interaction.

Different models have been developed in order to describe this new regime of the laser-matter interaction (cluster radius $R \ll$ laser wavelength $\lambda$ ). Inspired by the physics at the atomic and molecular level, the first one has been proposed by the group of C.K. Rhodes : the so-called "Coherent Electron Motion Model" (CEMM) [14]. In this model, the free electrons $(Z)$ from the ionized cluster oscillate coherently in the external laser field. They are considered as a quasi-particle possessing effective charge $Z e$ and mass $Z m$, increasing significantly the ionization efficiency in the collisions with intracluster ions and atoms. Limited to the treatment of small clusters ( $\lesssim 1000$ atoms), the CEMM reproduces the extremely high ionization levels observed in the x-ray measurements and predicts x-ray emission bursts as short as the laser duration.

In the case of larger clusters $\left(\sim 10^{6}\right.$ atoms $)$, the collisional processes are expected to dominate the lasercluster interaction, breaking the coherence of electron motion. In this regime, the "nano-plasma" model has been proposed by the group of T. Ditmire [15], including collisional mechanisms taken from classical laser-solid interactions. Basically, the interaction begins with the ionization of atoms in the external laser field. Then, the free electrons are accelerated and heated via the inverse bremsstrahlung process, leading to an additional collisional ionization. Finally, the cluster expansion is driven by both the hydrodynamic pressure and the Coulomb pressure (considering that some electrons have left the 
cluster). This model assumes instantaneously thermalized electrons. It has been developed considering a small homogeneous nano-plasma without any thermal or density gradients. The energy deposition is mainly driven by a huge laser field resonance inside the cluster, occurring during the cluster expansion when the electron density $n_{e}=3 n_{c}\left(n_{c}\right.$ is the critical density at $\left.\lambda\right)$. Two different time scales have been derived : the time $T_{\text {exp }}$ needed to reach this resonant condition (few 100 fs to ps) and the resonance duration $\delta t_{\text {res }}$ which has been estimated to be very short ( $\lesssim 10 \mathrm{fs})$. More recently, one-dimensional calculations have been proposed by the group of H.M. Milchberg $[16,17]$. They demonstrate that the laser energy deposition process is dominated by a large absorption in the density gradient close to the critical density $n_{c}$, similar to the classical resonant absorption in laser-solid interaction. The duration of the efficient laser absorption $\delta t_{a b s}$ is greatly increased in this description ( $\gtrsim 100 \mathrm{fs}$ ).

These models have been proposed to describe the dynamics of the cluster ionization, heating and expansion. Studies are still in progress and remain a great theoretical challenge. They need to be supported by experimental data, in order to confirm the mechanisms involved in the laser-cluster interaction and then to benchmark the existing codes. The lack of simulation is even greater concerning the $\mathrm{x}$-ray emission dynamics of irradiated clusters [18-21].

Time-integrated x-ray measurements are expected to shed some light on mechanisms involved in the first picoseconds of the interaction, when a large x-ray emission peak is expected from the clusters. In the present paper, we report an experimental study of the K-shell xray emission $(\sim 3 \mathrm{keV})$ of Argon clusters irradiated by intense femtosecond laser pulses. Time-integrated highresolution spectra are reported : i) as a function of various laser temporal parameters (from the ns down to the fs scale), ii) with both a linear and a circular polarization, and iii) as a function of the cluster size (varied from 180 to $350 \AA$ radius). The experimental observations are interpreted in term of the laser-cluster dynamics, leading to different time scales for the cluster expansion and the cluster heating. These results are then compared with the nano-plasma models.

\section{EXPERIMENTAL SET-UP}

Experiments have been performed with the CELIA $\mathrm{kHz}$ laser system [22]. Up to $4 \mathrm{~mJ}$ is delivered on target in 40 fs pulses FWHM (Full Width at Half Maximum) at $800 \mathrm{~nm}$ central wavelength. A quarter waveplate is placed in the laser beam in order to vary its polarization from linear to circular. The beam is focused into a $3.0 \pm 0.5 \mu \mathrm{m}$ spot (radius at $1 / e$ of the maximum intensity measured under vacuum). The energy measurements in the focal spot lead to a maximal intensity close to $(2.5 \pm 1.0) \times 10^{17} \mathrm{~W} / \mathrm{cm}^{2}$.

The temporal characteristics of the laser pulses have

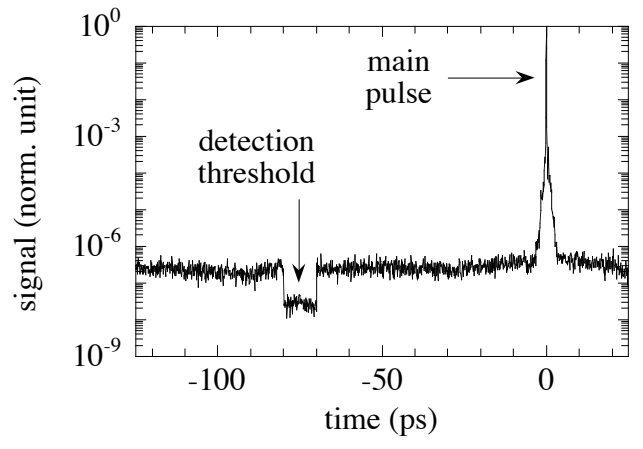

FIG. 1: Laser pulse temporal contrast measured with a third order autocorrelator (logarithmic scale). The detection threshold was determined blocking the laser beam during the scan between 80 and 70 ps before the main pulse.

been studied with special care since they could affect the dynamics of the laser-cluster interaction. The laser duration is measured using a second order autocorrelator. A minimum value of $40 \pm 5 \mathrm{fs}$ FWHM is obtained with the optimum compressor adjustment. The behavior versus the distance between the compressor gratings is fully calibrated, leading to the control of the laser pulse duration up to 2 ps within a $20 \%$ error bar. A third order autocorrelator trace is plotted in Fig. 1. A pedestal attributed to ASE (Amplified Spontaneous Emission) is measured at $(3 \pm 1) \times 10^{-7}$ of the main pulse, i.e. one order of magnitude above the estimated detection threshold. No pre-pulse is detected on this $100 \mathrm{ps}$ time scale. Due to the laser architecture, a pre-pulse is detected 4 ns before the main pulse. Its relative amplitude is observed with a photodiode. It is controlled with the adjustment of the Pockels cells delays, from less than $10^{-5}$ to $10^{-1}$.

The clusters are produced from the partial condensation of high pressure Argon expanding through a conical nozzle, designed to get a homogeneous cluster distribution in a $4 \mathrm{~mm}$ diameter jet. This study is performed with relatively large clusters $\left(\sim 10^{6}\right.$ atoms per cluster $)$ and the laser beam is focused $2 \mathrm{~mm}$ from the nozzle output. A previous study has led to the complete jet characterization in this configuration [23]. It has been supported by optical measurements and numerical simulations. The average cluster radii are given by $R=180,275$ and 350 $\AA$ for the corresponding Argon pressures $P_{0}=20,40$ and 60 bars. The standard deviation of the cluster radius distribution is expected to be limited by $\delta R / R<15$ $\%$. The total atomic density is in the range of $10^{19} \mathrm{~cm}^{-3}$ including the gas and the cluster phases. The condensation rate remains close to $25 \pm 10 \%$. The cluster jet is not operated continuously, in order to preserve an average residual pressure below a few $10^{-2}$ mbar inside the experimental chamber. It is opened for durations up to $40 \mathrm{~ms}$ each second. The time needed to get a stationary gas flow is expected to be below $1 \mathrm{~ms}$ [23], so that the cluster jet can be considered identical for each laser pulse during the $40 \mathrm{~ms}$ cluster jet interval. 


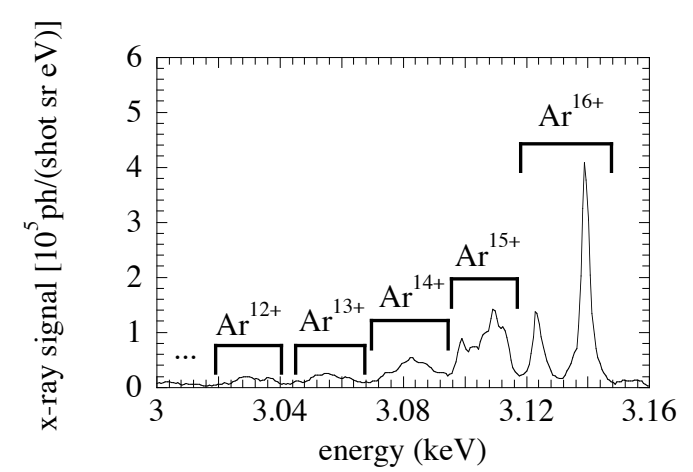

FIG. 2: K-shell x-ray spectrum of $350 \AA$ average radius Argon clusters irradiated by $500 \mathrm{fs}$ FWHM and $3.0 \mathrm{~mJ}$ laser pulses, corresponding to $1.6 \times 10^{16} \mathrm{~W} / \mathrm{cm}^{2}$ maximum intensity. Different lines are emitted from different charge state ions, up to $\mathrm{Ar}^{16+}$

\section{EXPERIMENTAL RESULTS}

In this dense jet regime, the x-ray emission has been observed to depend drastically on the position of focal plane in the cluster jet, indicating strong effects due to laser propagation. This point will be presented in detail in a forthcoming publication. In the present paper, the interaction is studied by focusing the laser on the edge of the cluster jet. The values of focal spot sizes, the maximum fluences and intensities are reported here as measured under vacuum.

\section{A. High resolution x-ray spectra}

The x-ray emission is spectrally resolved using a highresolution focusing spectrometer developed in our group in collaboration with the group of A. Ya. Faenov from VNIIFTRI, Mendeleevo, Russia [24, 25]. The timeintegrated spectra are measured in the range from 2.9 to $3.3 \mathrm{keV}$ in the fourth order of a Mica crystal, and recorded on a 16 bit x-ray Charge Coupled Device (CCD) camera. The spectral resolution is $2 \mathrm{eV}$. The $\mathrm{x}$-ray spectrometer efficiency is calibrated within a $20 \%$ error bar. The spectral dispersion is calibrated from tabulated resonance lines $\mathrm{He}_{\alpha}$ (and its intercombination line), $\mathrm{He}_{\beta}$, $\mathrm{He}_{\gamma}$ and $\mathrm{He}_{\delta}$, respectively corresponding to transitions from the $1 s 2 p(1 s 2 s), 1 s 3 p, 1 s 4 p$ and $1 s 5 p$ levels to the $1 s^{2}$ level [38]

Each x-ray spectrum is the result of an accumulation of a few tens of thousand shots. The figure 2 presents the spectrum obtained from $350 \AA$ average radius Argon clusters irradiated by $500 \mathrm{fs}, 3.0 \mathrm{~mJ}$ laser pulses. The estimated maximum intensity is $1.6 \times 10^{16} \mathrm{~W} / \mathrm{cm}^{2}$ and the relative amplitude of the 4 ns pre-pulse is $2 \times 10^{-6}$ (cf. Section IIIB). Different lines are observed, a few of them arise from the fifth order diffraction of the Mica crystal.
We focus on the $\mathrm{He}_{\alpha}$ line and its satellites, since they consist of transitions from ions of different charge states, emitted through well separated and identified lines. For clarity reason, they are only presented on Figure 2 in the range from 3.00 to $3.16 \mathrm{keV}$. The emission is observed from very highly charged Argon ions, from $\mathrm{Ar}^{12+}$ up to $\mathrm{Ar}^{16+}$. No emission is observed in the "cold" $\mathrm{K}_{\alpha}$ line, corresponding to $\mathrm{Ar}^{8+}$ or less. This could indicate that the neutral atoms (or the ions without vacancy in the Lshell) are no longer present in the cluster when the x-ray emission occurs.

The observed K-shell emission requires a significant fraction of electrons with an energy typically $>4 \mathrm{keV}[21]$ to induce $\mathrm{K}$-shell vacancies. It corresponds in fact to the maximum energy in the collisionless motion of an electron in a linearly polarized laser field with radiation intensity $>3.3 \times 10^{16} \mathrm{~W} / \mathrm{cm}^{2}$. Circular polarization with this intensity would give rise to a constant orbital energy of the same value $4 \mathrm{keV}$. Measurements are performed with both a linear and a circular laser polarization. The x-ray signal remains the same, confirming previously published results [26]. Even the line shapes and amplitudes remain unchanged, indicating that the heating is dominated by collisional processes in this regime of large clusters, as assumed in the "nano-plasma" models.

The x-ray spectra are observed through different angles between $20^{\circ}$ and $160^{\circ}$ from the incident laser beam, without any significant modification. This suggests an isotropy over $4 \pi \mathrm{sr}$. This is expected from the spontaneous emission of a nano-plasma in the x-ray range of interest for which re-absorption could be neglected. As presented in Fig. 2, the emission in $\mathrm{He}_{\alpha}$ line reaches $(4.2 \pm 0.5) \times 10^{5}$ photons $/(\mathrm{sr}$ shot $\mathrm{eV})$. This corresponds to $(2.0 \pm 0.5) \times 10^{8} \mathrm{~K}$-shell photons per shot in $4 \pi \mathrm{sr}$, leading to $(3 \pm 1) \times 10^{-5}$ conversion efficiency from the laser energy to the Ar K-shell emission $(\sim 3 \mathrm{keV})$. In the next sections, the x-ray emission in $\mathrm{He}_{\alpha}$ is used in order to quantify the x-ray intensity as a function of the different laser and cluster parameters.

\section{B. Influence of the laser temporal contrast}

The x-ray spectra are studied as a function of the $4 \mathrm{~ns}$ pre-pulse laser temporal contrast. The figure 3 reports measurements performed with a 300 fs and $3.0 \times 10^{16}$ $\mathrm{W} / \mathrm{cm}^{2}$ main laser pulse irradiating $275 \AA$ average radius clusters. The intensity of the 4 ns pre-pulse is increased from less than $8 \times 10^{11}$ (limited by detection threshold) to $5 \times 10^{15} \mathrm{~W} / \mathrm{cm}^{2}$. The $\mathrm{x}$-ray signal is observed to decrease by more than one order of magnitude when the pre-pulse intensity exceeds a threshold between 0.8 and $4.0 \times 10^{14}$ $\mathrm{W} / \mathrm{cm}^{2}$. This feature suggests that the clusters could be ionized by the pre-pulse and then expand in less than 4 ns. The main laser pulse would then interact with a gas density target, that could explain the drastic reduction of the x-ray production. The relatively slow decrease observed in Fig. 3 should be attributed to the spatial 


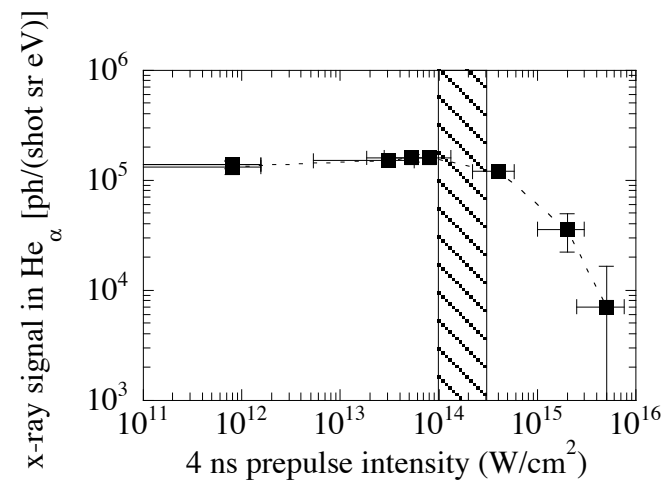

FIG. 3: X-ray emission in $\mathrm{He}_{\alpha}$ line measured as a function of the 4 ns laser pre-pulse intensity. $275 \pm 40 \AA$ radius Ar clusters are irradiated by $300 \mathrm{fs}$ FWHM and $3.0 \pm 0.2 \mathrm{~mJ}$ laser pulses, corresponding to a maximum intensity of $(3.0 \pm 0.6) \times 10^{16}$ $\mathrm{W} / \mathrm{cm}^{2}$. The hatched area indicates the intensity range of a $300 \mathrm{fs}$ laser pulse leading to the single ionization of Argon with a probability between 10 to $90 \%$.

integration over the focal volume.

Calculations based on the ADK rate [27] are performed in order to deduce the probability of Argon single ionization with 300 fs FWHM laser pulses of variable maximum intensities. The intensity range leading to a probability between 10 and $90 \%$ is shown in Fig. 3. It presents a good agreement with the observed threshold. Two conclusions could be drawn from these measurements. First, within the experimental uncertainty, they validate the assumption made in the nano-plasma models that the first free electrons are expelled via the tunnel ionization. Second, they confirm that the dynamics of the ionized cluster takes place over a time scale shorter than the nanosecond.

In order to prevent any modifications of the clusters before the interaction with the main laser pulse, all the other results presented in this paper are obtained with a 4 ns pre-pulse whose relative amplitude is lower than $2 \times 10^{-6}$ (limited by the detection threshold). As a picosecond pre-pulse has been previously suspected to be important in the generation of the x-ray spectra [19, 28], it is studied with a special care, using the third order autocorrelator. A measurement is plotted in Fig. 4 with the same parameters for the main laser pulse as in Fig. 3. The intensity range for a single ionization of Argon calculated between 10 to $90 \%$ is reported. This figure clearly demonstrates that no pre-pulse is expected to pre-ionize the clusters, providing experimental conditions as clean as possible for comparison with the models.

\section{Study with varying laser pulse duration}

The x-ray spectra are measured for various pulse durations from 40 fs to 2 ps FWHM. The x-ray signal in $\mathrm{He}_{\alpha}$ is plotted in Fig. 5, when Argon clusters with $350 \AA$ average radius are irradiated by $3.0 \mathrm{~mJ}$ laser pulses. The

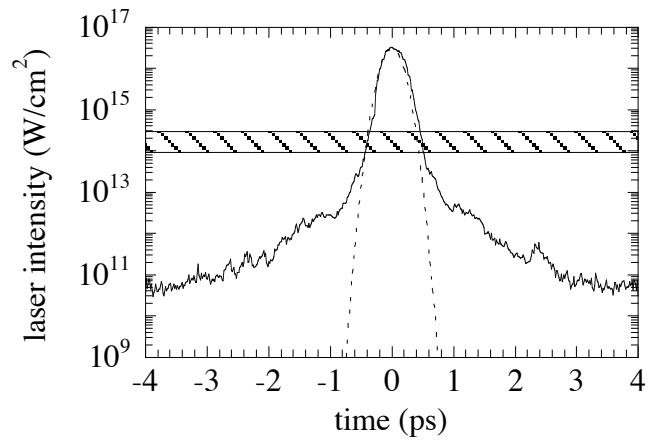

FIG. 4: Detail of the laser pulse temporal contrast measured with a third order autocorrelator for a 300 fs FWHM and $3.0 \pm 0.2 \mathrm{~mJ}$ main laser pulse, corresponding to a maximum intensity of $(3.0 \pm 0.6) \times 10^{16} \mathrm{~W} / \mathrm{cm}^{2}$. The hatched area indicates the intensity range leading to the single ionization of Argon with a probability between 10 to $90 \%$.

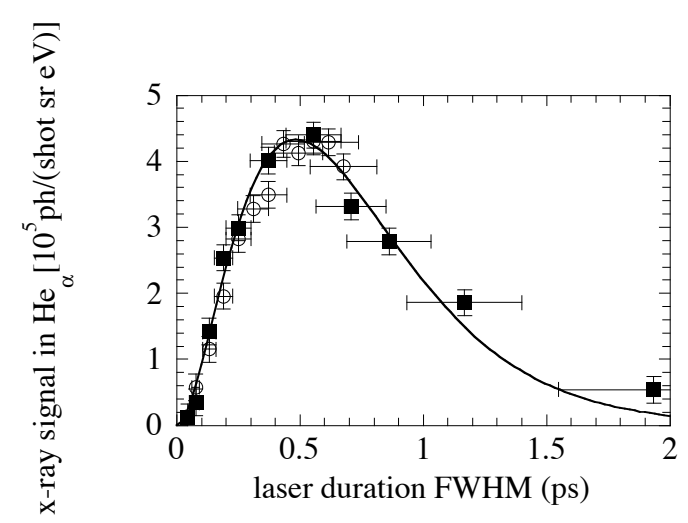

FIG. 5: X-ray emission in $\mathrm{He}_{\alpha}$ line measured as a function of the laser duration. $350 \AA$ radius Ar clusters are irradiated by $3.0 \mathrm{~mJ}$ laser pulses, corresponding to a maximal fluence on target of $8.0 \times 10^{3} \mathrm{~J} / \mathrm{cm}^{2}$. Empty circles : negative chirp. Full squares : positive chirp.

fluence is maintained at $8.0 \times 10^{3} \mathrm{~J} / \mathrm{cm}^{2}$ on the target, while the maximum intensity is decreased from $2.0 \times 10^{17}$ $\mathrm{W} / \mathrm{cm}^{2}$ (at $40 \mathrm{fs}$ ) to $4.0 \times 10^{15} \mathrm{~W} / \mathrm{cm}^{2}$ (at $2 \mathrm{ps}$ ). Measurements are reported with a positive and a negative chirp without clear distinction between them [29]. This can be understood within the frame of the nano-plasma models. The dynamics of the interaction is predicted to be mainly driven by the temporal distribution of the laser energy. In our case, the laser spectrum is symmetric so that the sign of the chirp should just modify the order of appearance of the frequencies inside the laser envelope without changing the pulse shape.

The x-ray intensity is observed to reach a maximum for an optimum laser duration $T_{\text {opt }}$. The experimental data are fitted by an arbitrary function proportional to $\tau^{2} \exp \left(-2 \tau / T_{\text {opt }}\right)$, where $\tau$ is laser duration. An optimum duration $T_{\text {opt }}=500 \pm 50$ fs is deduced from the fit. This result indicates a regime far from the interaction 


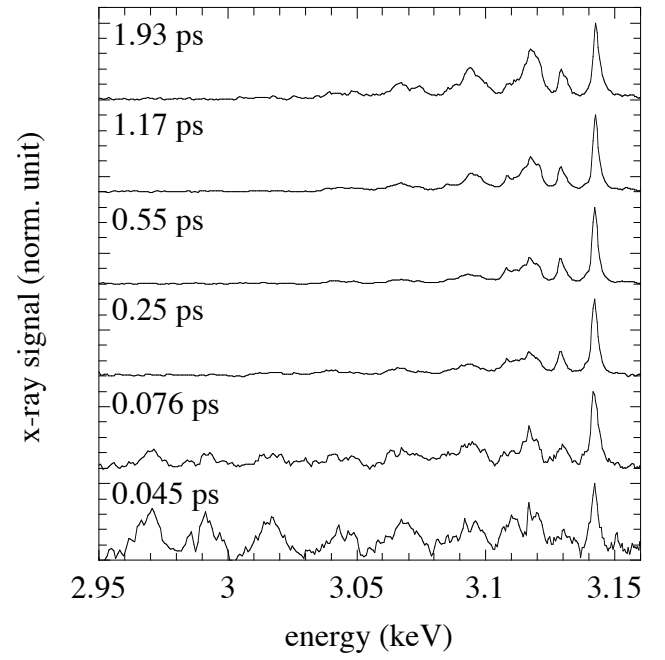

FIG. 6: K-shell x-ray spectra measured as a function of the laser duration. Argon clusters with $350 \AA$ average radius are irradiated by $3.0 \mathrm{~mJ}$ laser pulses, corresponding to a maximum fluence of $8.0 \times 10^{3} \mathrm{~J} / \mathrm{cm}^{2}$. The laser pulse durations FWHM are indicated on the corresponding normalized spectra.

with a single atom where the electron energy increases with the laser intensity. Similar features have been previously observed in absorption experiments [30], indicating that a characteristic time should be necessary to optimize the energy deposition in the clusters.

The optimum duration $T_{o p t}$ is estimated for the x-ray emission through the $\mathrm{He}_{\alpha}$ line. Similar results are obtained for the other lines. Nevertheless, the spectra aspects are observed to strongly depend on the laser duration, as shown in Fig. 6. The general aspect is similar to the one presented in Fig. 2 for a laser duration close to or higher than $T_{o p t}$. In the special case of the shortest durations (i.e. $<100 \mathrm{fs}$ ), the contribution from the other lines is higher relative to the $\mathrm{He}_{\alpha}$ line. This demonstrates a larger distribution of the ionic charge states ranging from $\mathrm{Ar}^{15+}$ down to $\mathrm{Ar}^{9+}$. This suggests that a certain pulse duration is required to produce the highest charge states.

The same experiment is performed with different sizes of clusters (from 180 to $350 \AA$ average radius). The data are plotted in Fig. 7. The same general behavior is observed as a function of the laser pulse duration. The optimum duration $T_{\text {opt }}$ increases with the cluster size. The influence of the laser energy is also studied. The figure 8 presents the data obtained with $275 \AA$ average radius clusters irradiated with different maximum laser fluences. The same behavior is again observed. As expected (cf. e.g. [21]), the x-ray emission is found to increase with the laser energy. The optimum duration is estimated to slightly increase when the laser energy decreases, as will be discussed in the section IV A.

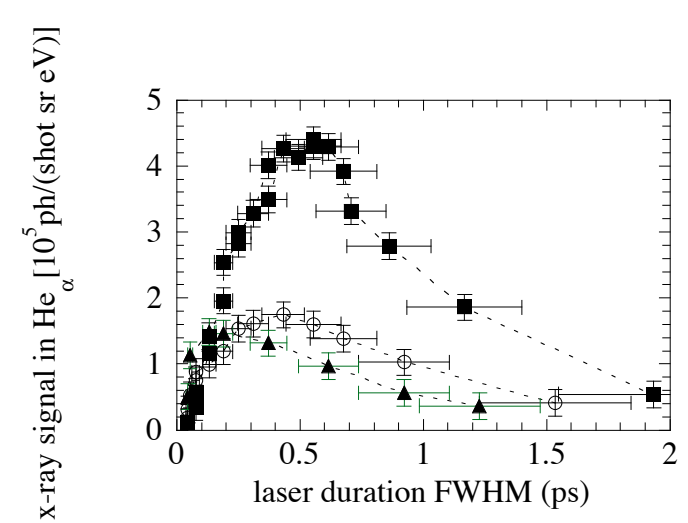

FIG. 7: X-ray emission in the $\mathrm{He}_{\alpha}$ line measured as a function of the laser duration. Ar clusters with different sizes are irradiated by $3.0 \mathrm{~mJ}$ laser pulses, i.e. a maximum fluence on target of $8.0 \times 10^{3} \mathrm{~J} / \mathrm{cm}^{2}$. Full triangles : clusters with 180 $\AA$ average radius are irradiated. Empty circles : $275 \AA$. Full squares : $350 \AA$.

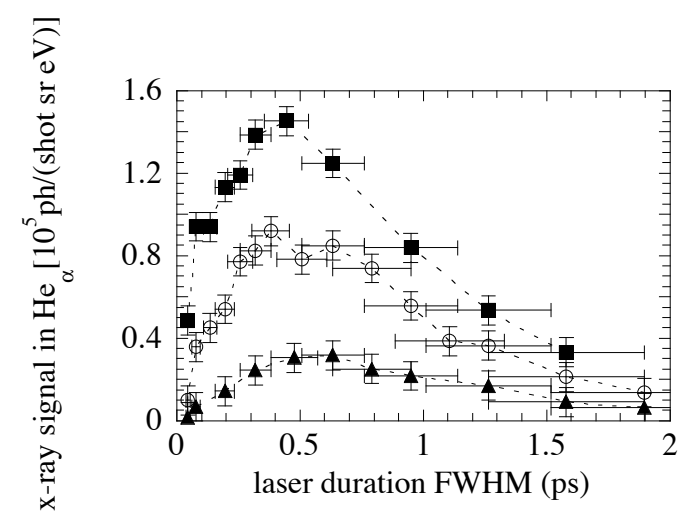

FIG. 8: X-ray emission in the $\mathrm{He}_{\alpha}$ line measured from clusters with $275 \AA$ average radius, as a function of the laser duration. Full triangles : $1.8 \mathrm{~mJ}$ in the laser pulse corresponding to a maximum fluence of $4.8 \times 10^{3} \mathrm{~J} / \mathrm{cm}^{2}$. Empty circles : $2.8 \mathrm{~mJ}$, i.e. $7.5 \times 10^{3} \mathrm{~J} / \mathrm{cm}^{2}$. Full squares : $3.8 \mathrm{~mJ}$, i.e. $1.0 \times 10^{4}$ $\mathrm{J} / \mathrm{cm}^{2}$.

\section{Experiments with two laser pulses}

Experiments are performed irradiating the clusters with two identical $40 \pm 5$ fs FWHM laser pulses. They are produced with a Michelson-type optical set-up. Due to the focusing, their durations are estimated to become $45 \pm 15$ fs on the target. The delay between the two pulses is varied with a motorized translation stage. Several diaphragms are inserted on one of the two beams in order to vary the irradiation fluence of the first pulse on the target.

The x-ray measurements are plotted in Fig. 9 for three different fluences of the first pulse as a function of the second pulse delay. The general features observed are : i) the signal is close to or below the detection thresh- 


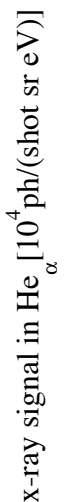

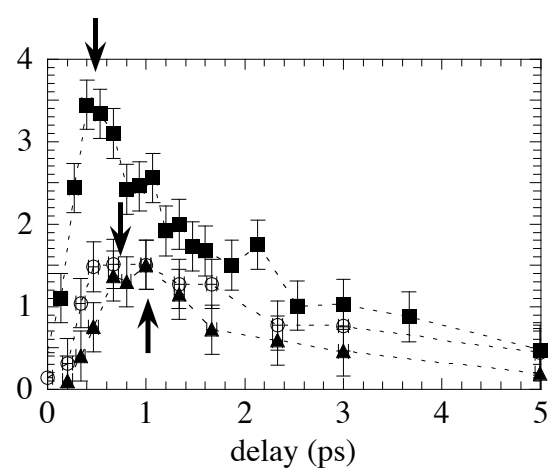

FIG. 9: X-ray emission in the $\mathrm{He}_{\alpha}$ line measured as a function of the delay between two $45 \pm 15$ fs FWHM laser pulses irradiating $350 \AA$ average radius clusters. Full triangles : with $1.0 \times 10^{3} \mathrm{~J} / \mathrm{cm}^{2}$ in the first pulse. Empty circles : $1.9 \times 10^{3}$ $\mathrm{J} / \mathrm{cm}^{2}$. Full squares : $3.2 \times 10^{3} \mathrm{~J} / \mathrm{cm}^{2}$. The fluence of the second pulse is maintained at $3.2 \times 10^{3} \mathrm{~J} / \mathrm{cm}^{2}$. The optimum delays $T_{\max }$ are indicated with arrows.

old when the two pulses are overlapped in time, ii) it increases rapidly to reach a maximum value for an optimum delay $T_{\max }$, iii) then it decreases very slowly : a signal is still detected with a delay larger than 5 picoseconds. $T_{\max }$ is estimated at the peak of the rapid increase as indicated by the arrows in Fig. 9. Technically, the experimental points up to the peak are fitted with a gaussian function and its maximum is used to assign a value to $T_{\max }$. Such a procedure results in large error bars for the estimated $T_{\max }$ values.

With an optimum delay, the spectra are significantly different compared to the one obtained with a single pulse of optimum duration as illustrated in Fig. 10. A larger contribution of the lines from low charge state ions is measured : down to $\mathrm{Ar}^{9+}$, similar to the spectrum with a 45 fs single laser pulse.

\section{DISCUSSION}

The measurements reported in Section III A as a function of the laser polarization suggest that the motion of the electrons is dominated by collisional processes. As a consequence, the CEMM is not considered for the data analysis. Instead, the nano-plasma models which are more adapted to this large cluster regime (more than $10^{6}$ atoms per cluster) are considered. The experiment presented in the section IIIB as a function of the $4 \mathrm{~ns}$ pre-pulse intensity confirms that tunnel ionization is the first mechanism involved in the laser-cluster interaction.

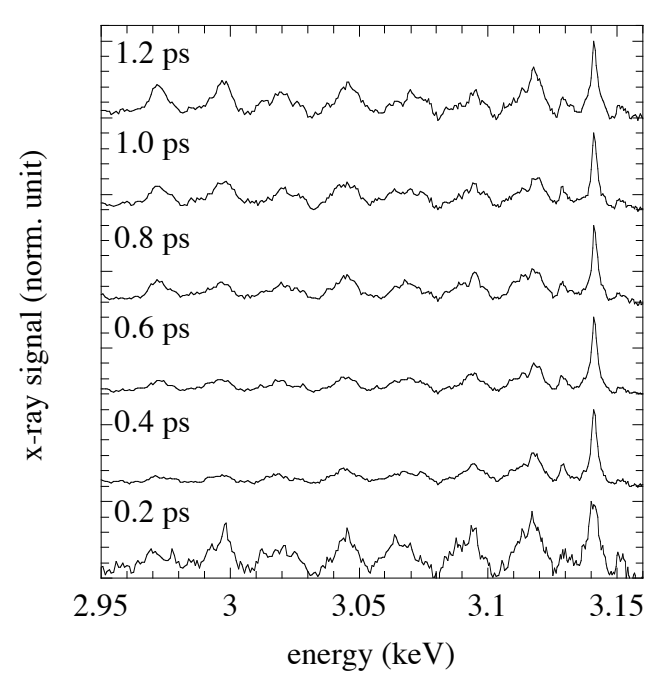

FIG. 10: K-shell x-ray spectra measured as a function of the delay between two $45 \pm 15$ fs FWHM laser pulses irradiating Argon clusters with $350 \AA$ average radius. The maximum fluence of the two pulses on target is set to $3.2 \times 10^{3} \mathrm{~J} / \mathrm{cm}^{2}$. The delays between the pulses are indicated on the corresponding normalized spectra.

\section{A. Dynamics of absorption and heating}

The K-shell x-ray spectra are the results of inner shell vacancies that should be generated by energetic electrons. In that sense, the observation of intense x-ray emission should be associated to an efficient coupling from the laser energy to the free electrons of the irradiated clusters. Considering this, the measurements reported in Fig. 7 confirm previous observations of an optimum duration for a single laser pulse to be efficiently absorbed in a cluster target [30]. In this reference, the optimum duration is interpreted within the "nano-plasma" model in terms of an absorption resonance encountered when the free electron density in the cluster is close to $3 n_{c}$. In the one-dimensional model extension [16], this resonance density $n_{\text {res }}$ is expected to be the critical density $n_{c}$. The time needed for the electron density to decrease from its initial value $n_{0}$ down to $n_{\text {res }}$ is given by Eq. 1 as a function of the average radius $R$ of the clusters [15]. $C_{s}$ designates the ion acoustic velocity that is proportional to the square root of the energy initially deposited in the electrons.

$$
T_{\exp }=\frac{R}{C_{s}}\left(\frac{n_{0}}{n_{r e s}}\right)^{\frac{1}{3}}
$$

In the present paper, the optimum durations $T_{\text {opt }}$ are determined from single pulse duration scans. They are reported in Table I. Two power scaling laws are derived, i) as a function of the characterized average radius $R$ of the clusters (cf. Eq. 2), and as a function of the laser maximal fluence $F$ on the target as estimated under vacuum (cf. Eq. 3). A linear dependence of $T_{o p t}$ on the 
TABLE I: Optimum laser duration $T_{\text {opt }}$ (FWHM) for the xray production in the $\mathrm{He}_{\alpha}$ line with a single pulse. The values are deduced from the experimental data as described in the section III C, as a function of the cluster average radius $R$ and of the laser energy $E$. The maximum laser fluences $F\left(\mathrm{~J} / \mathrm{cm}^{2}\right)$ on the target can be estimated under vacuum, by multiplying $E(\mathrm{~mJ})$ by $(3.5 \pm 1.4) \times 10^{3}$.

\begin{tabular}{ccc}
\hline \hline$E(\mathrm{~mJ})$ & $R(\AA)$ & $T_{\text {opt }}(\mathrm{ps})$ \\
\hline $3.0 \pm 0.2$ & $180 \pm 25$ & $0.25 \pm 0.05$ \\
$3.0 \pm 0.2$ & $275 \pm 40$ & $0.40 \pm 0.07$ \\
$3.0 \pm 0.2$ & $350 \pm 50$ & $0.50 \pm 0.05$ \\
& & \\
$1.8 \pm 0.2$ & $275 \pm 40$ & $0.58 \pm 0.05$ \\
$2.8 \pm 0.2$ & $275 \pm 40$ & $0.48 \pm 0.06$ \\
$3.8 \pm 0.2$ & $275 \pm 40$ & $0.42 \pm 0.07$ \\
\hline \hline
\end{tabular}

cluster average radius $R$ is observed, in good agreement with Eq. 1 derived from the "nano-plasma" models. This would confirm that the laser pulse has to be as long as the expansion time $T_{\exp }$ needed for the clusters to reach the characteristic density $n_{\text {res }}$ for efficient absorption and electron heating. If we assume that the laser energy deposited in the cluster is proportional to the incident laser energy, then the dependence on the laser energy should be considered also in good agreement with this picture.

$$
T_{o p t} \propto R^{1.05 \pm 0.15}
$$

$$
T_{\text {opt }} \propto F^{-0.45 \pm 0.15}
$$

Sub-picosecond times are derived for the cluster expansion from these single pulse experiments. But they can not resolve the duration $\delta t_{a b s}$ of the efficient absorption process. Moreover, Eq. 1 assumes that the cluster expansion starts from an instantaneous initial heating of the electrons, which is a priori far from the reality with picosecond laser pulses. The experiments with two delayed 45 fs FWHM laser pulses are more suitable to extract the temporal parameters of the dynamics of the laser-cluster interaction. The first pulse is responsible for the initial heating of the cluster and initiates its expansion. The $\mathrm{x}$-ray emission induced by this first pulse alone is observed to be close to the detection threshold. Then the $\mathrm{x}$-ray emission measured when the cluster is irradiated with the two delayed pulses is mainly due to the efficient heating of the cluster by the second pulse. The delay $T_{\max }$ that is observed to maximize the x-ray emission, is reported in Table II as a function of the laser energy in the first pulse for clusters with $350 \AA$ average radius. The delays obtained are very similar to the values of $T_{o p t}$ derived from the single pulse experiments. A power scaling law is deduced as a function of the maximum fluence $F$ of the first pulse on target (cf. Eq. 4).

$$
T_{\max } \propto F^{-0.60 \pm 0.25}
$$

TABLE II: Optimum delay $T_{\max }$ between the two $45 \mathrm{fs}$ FWHM laser pulses for the x-ray production in the $\mathrm{He}_{\alpha}$ line. The values are deduced from the experimental data as a function of the laser energy $E$ in the first laser pulse. The corresponding maximum laser fluences $F$ on clusters with $350 \AA$ average radius are indicated, as estimated under vacuum.

\begin{tabular}{ccc}
\hline \hline$E(\mathrm{~mJ})$ & $F\left(\mathrm{~J} / \mathrm{cm}^{2}\right)$ & $T_{\max }(\mathrm{ps})$ \\
\hline $0.45 \pm 0.05$ & $(1.0 \pm 0.4) \times 10^{3}$ & $1.00 \pm 0.25$ \\
$0.70 \pm 0.05$ & $(1.9 \pm 0.7) \times 10^{3}$ & $0.75 \pm 0.20$ \\
$0.90 \pm 0.05$ & $(3.2 \pm 1.3) \times 10^{3}$ & $0.50 \pm 0.15$ \\
\hline \hline
\end{tabular}

Within the large uncertainty, and assuming that the initial heating of the cluster is proportional to the laser energy in the first pulse, one could observe a reasonable agreement with the nano-plasma models prediction (i.e. agreement between Eq. 4 and Eq. 1). This confirms that a characteristic electron density $n_{\text {res }}$ is required inside the cluster to efficiently absorb the laser energy in the heating of the free electrons, as proposed in both references $[15,16]$. The measurements do not provide the determination of $n_{\text {res }}$ between the two different values proposed ( $3 n_{c}$ in Ref. [15] and $n_{c}$ in Ref. [16]). However, the experimental data plotted in Fig. 9 demonstrate that the electron heating by the second laser pulse could be efficient within picoseconds after the irradiation with the first pulse. This result is clearly in contradiction with the very short resonant duration $\left(\delta t_{\text {res }} \sim 10 \mathrm{fs}\right)$ predicted by the original "nano-plasma" model [15]. The one dimensional extension of the model could be more appropriate to describe the laser absorption and the dynamics of the cluster heating [16]. Other effects that could contribute to enlarge the observed $\delta t_{a b s}$ are i) the distribution of the cluster size in the jet; ii) the distribution of the laser fluence on the clusters in the interaction volume.

\section{B. X-ray spectra lines analysis}

The K-shell x-ray spectra measured indicate the presence of high ionic charge states in the emitting plasma. This observation has been previously reported in several papers $[18,21,28]$ with similar laser and cluster parameters. The ionization state inferred from the x-ray lines emitted is observed to increase when the duration of a single laser pulse approaches the optimal value $T_{\text {opt }}$ (cf. Fig. 6). In this case, the x-ray spectra are dominated by the lines emitted by $\mathrm{Ar}^{15+}$ and $\mathrm{Ar}^{16+}$ ions. This indicates that a certain time (a few hundreds of femtoseconds) is necessary to reach the highest ionization states, as expected from a model of sequential ionization. The experiments with the two delayed short pulses demonstrate that the $\mathrm{x}$-ray spectra are not so strongly modified with the delay (cf. Fig. 10). They show similarities to the one recorded using a single 45 fs pulse, i.e. suggesting a large distribution of emitting ions from $\mathrm{Ar}^{9+}$ to $\mathrm{Ar}^{16+}$. The most probable scenario that could be con- 


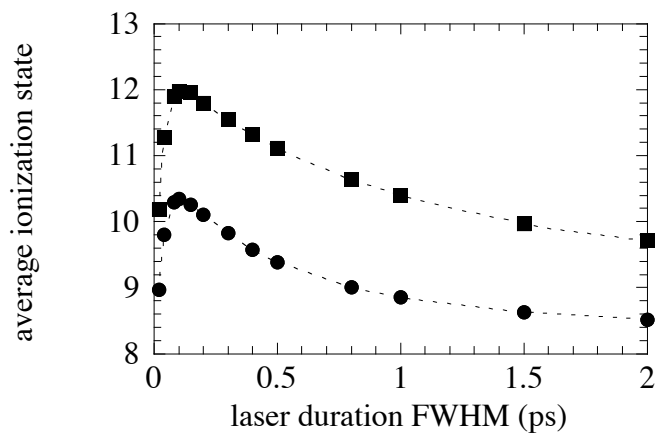

FIG. 11: Average ionic charge state $\langle Z\rangle$ deduced from calculations for different laser durations and cluster sizes. The maximum fluence is set to $8.0 \times 10^{3} \mathrm{~J} / \mathrm{cm}^{2}$ on the target, as estimated under vacuum during the experiments. Full circles : calculations for $180 \AA$ clusters. Full squares : calculations for $350 \AA$ clusters.

cluded from this result is that the ionization is mainly achieved by the first pulse interacting with a high density nanoplasma. The second pulse would heat the lower density plasma, but it would be too short to drastically increase the ionization.

The major aspect that is still to be understood is the presence, and in many cases the dominance of $\mathrm{Ar}^{16+}$ ions. Within the framework of the nano-plasma model [15], the production of the high charge states is ascribed to the collisional ionization by the energetic electrons driven by the giant laser field resonance occurring when the electron density decreases to $n_{e}=3 n_{c}$ during the plasma expansion. In order to investigate this issue more in detail, a computer code is developed including the mechanisms proposed in the reference [15]. In integrating the equations for the temporal evolution of the nano-plasma, a major problem occurs at the resonance for $n_{e}=3 n_{c}$, where the laser field and the plasma heating rate grow to unreasonably high values. This difficulty has been circumscribed by Megi et al. [31] introducing collisions between the electrons and the cluster surface at a rate $s=v / R$, where $v^{2}=\left(v_{t h}^{2}+v_{q}^{2}\right)$ is the effective electron velocity. It takes into account both the thermal velocity $v_{t h}$ and the quiver velocity $v_{q}$. These collisions dominate the electron heating process near the resonance, where $v$ is high, and suppress the singularity in the temporal evolution of the nano-plasma. Although quite arbitrary, we have adopted this mechanism in our computer code, and obtain stable solutions with finite values for the electronion collision frequency and the electron temperature near the resonance.

Figure 11 presents the average ionic charge state reached at the end of the laser pulse, computed by our code as a function of the pulse duration with constant fluence $8.0 \times 10^{3} \mathrm{~J} / \mathrm{cm}^{2}$ on the target, and for two different cluster sizes. The optimum pulse duration deduced for the production of high charge states is shorter $(\sim 100$ fs) than the experimentally observed value. The model fails to predict ionic charge states as high as $\mathrm{Ar}^{16+}$. The highest charge state reached in the computer simulation is $\mathrm{Ar}^{14+}$ (less than $10 \%$ of the ions), the majority ion being $\mathrm{Ar}^{12+}$ when using 100 fs laser pulses interacting with $350 \AA$ argon clusters.

Additional mechanisms have to be considered in order to reproduce the observed charge states. The collisional ionization is evaluated using the Lotz cross section [32], which is an empirical formula valid for a binary impact between an electron and an isolated ion. Inside a cluster the ionization rate could be enhanced by Coulomb-barrier lowering due to the neighboring ions [33], by screening effects of the Coulomb interactions at high electron density [34], and by coherent multi-electron motion [14]. In the 1D model of Milchberg et al. [16], the laser energy is deposited in the high density gradient close to the critical density $n_{c}$, that moves inwards during the laser-cluster interaction. This could have the effect of greatly increasing the efficiency and the duration of the laser-plasma coupling, and locally producing high charge states.

Recently a very simplified model of the interaction has been proposed by Smirnov and Krainov [35]. The ionization of the cluster ions ("inner ionization") is caused by the combination of the laser field and the static radial field resulting from a fraction of the free electrons having left the cluster ("outer ionization"). The coherent motion of these quasi-free electrons could give rise to additional effects, such as the quasi-particle enhanced ionization [14], or the quasiresonance ionization mechanism found in the classical trajectory studies performed for smaller clusters $\left(\sim 10^{3}\right.$ ions) [36]. The latter effect is related to the motion of the unbound electrons in the entire cluster, and could lead to an "outer ionization" significantly higher than predicted by Ditmire's formula [15], that would in turn increase the intra-cluster fields and the ionic charge states by barrier-suppression. The "ionization ignition" mechanism, first proposed by RosePetruck et al. [37] should be considered together with these effects. More work, both experimental and theoretical, is clearly needed in order to gain a better understanding of the various processes taking place in the dynamics of the laser-cluster interaction.

\section{CONCLUSION}

Large Argon clusters (average radius ranging from 180 to $350 \AA$ as previously measured [23]) are irradiated by intense femtosecond laser pulses (up to a few $10^{17}$ $\left.\mathrm{W} / \mathrm{cm}^{2}\right)$. K-shell $\mathrm{x}$-ray spectra are registered as a function of the size of the clusters, the laser energy, its polarization and different laser temporal parameters (the nanosecond contrast, the single pulse duration and the delay between two pulses). The study varying the polarization shows that the interaction is dominated by the collisions in this regime. The laser intensity threshold for the destruction of the clusters is observed to correspond to the ionization threshold through ADK calcula- 
tions [27], indicating that tunnel ionization should be the first mechanism involved in the intense laser - clusters interaction. The study as a function of the duration of a single laser pulse demonstrates an optimal duration for the x-ray production, that linearly depends on the clusters size and ranges from 250 to 580 fs. With lower laser energies, a delay between two laser pulses is measured to optimize the x-ray production in a similar range from 500 fs to 1 ps. These results suggest that an optimal average electron density has to be reached through expansion for the laser to efficiently heat the clusters. The experimental data are compared to the nano-plasma model [15]. This model predicts an absorption resonance when the density is close to $3 n_{c}$ for a very short time inter$\operatorname{val}\left(\delta t_{r e s} \sim 10 \mathrm{fs}\right)$ that is not observed in the experiment with the two laser pulses. The one-dimensional extension of the nano-plasma model [16] could contribute to the description of the experimental results. The heating is then expected to occur mainly at the critical density $n_{c}$ which would exist in the density gradients for a longer time, comparable with the few picoseconds observed. Simulations are performed, clearly indicating that additional mechanisms should be considered to reproduce the high ionization states observed (up to $\mathrm{Ar}^{16+}$ ).

\section{Acknowledgments}

The authors would like to thank Eric Mevel and Eric Constant for helpful discussions about ADK ionization calculations ; the staff from Groupe de Physique des Solides (Jussieu, Paris, France) and CEA/DSM/DRECAM/SPAM (Saclay, France) for their help in the x-ray spectrometer absolute calibration. We gratefully acknowledge computational facilities provided by the intensive calculation pole M3PECMESOCENTRE of the University Bordeaux I-DRIMM. It is also a great pleasure to acknowledge G. Darpentigny for his precious help on the laser, as well as A. Le Goff for his technical assistance. This work is partially supported by the Fond Européen de Développement Economique Régional and the Conseil Régional d'Aquitaine.
[1] J. W. G. Tisch, in Atoms, Solids and Plasmas in SuperIntense Laser Fields, edited by D. Batani, C. J. Joachain, S. Martellucci, and A. N. Chester (Plenum, New York, 2001), pp. 99-118.

[2] A. McPherson, T. Luk, B. Thompson, A. Borisov, O. Shiryaev, X. Chen, K. Boyer, and C. Rhodes, Phys. Rev. Lett. 72, 1810 (1994).

[3] T. Ditmire, T. Donnelly, R. W. Falcone, and M. D. Perry, Phys. Rev. Lett. 75, 3122 (1995).

[4] Y. L. Shao, T. Ditmire, J. W. G. Tisch, J. P. M. E. Springate, and M. H. R. Hutchinson, Phys. Rev. Lett. 77, 3343 (1996).

[5] T. Ditmire, J. W. G. Tish, E. Springate, M. B. Mason, N. Hay, R. A. Smith, J. Marangos, and M. H. R. Hutchinson, Nature 386, 54 (1997).

[6] M. Lezius, S. Dobosz, D. Normand, and M. Schmidt, Phys. Rev. Lett. 80, 261 (1998).

[7] E. Springate, S. A. Aseyev, S. Zamith, and M. J. J. Vrakking, Phys. Rev. A 68, 053201 (2003).

[8] T. Ditmire, J. Zweiback, V. P. Yanovsky, T. E. Cowan, G. Hays, and K. B. Wharton, Nature 398, 489 (1999).

[9] T. Ditmire, R. A. Smith, J. W. G. Tisch, and M. H. R. Hutchinson, Phys. Rev. Lett. 78, 3121 (1997).

[10] G. D. Kubiak, L. J. Bernardez, K. D. Krenz, D. J. O'Connell, R. Gutowski, and A. M. Todd, OSA Trends Opt. Photonics Ser. 4, 66 (1996).

[11] C. Rischel, A. Rousse, I. Uschmann, P. A. Albouy, J. P. Geindre, P. Audebert, J. C. Gauthier, E. Foerster, J. L. Martin, and A. Antonetti, Nature 390, 490 (1997).

[12] J. Larsson and A. Sjogren, Rev. Sc. Instrum. 70, 2253 (1999).

[13] T. Shiraishi, M. Mori, and K. Kondo, Phys. Rev. A 65, 045201 (2002).

[14] K. Boyer, B. Thompson, A. McPherson, and C. Rhodes, J. Phys. B: At. Mol. Opt. Phys. 27, 4373 (1994).
[15] T. Ditmire, T. Donnelly, A. M. Rubenchik, R. W. Falcone, and M. D. Perry, Phys. Rev. A 53, 3379 (1996).

[16] H. M. Milchberg, S. J. McNaught, and E. Parra, Phys. Rev. E 64, 056402 (2001).

[17] K. Y. Kim, I. Alexeev, E. Parra, and H. M. Milchberg, Phys. Rev. Lett. 90, 023401 (2003).

[18] G. C. Junkel-Vives, J. Abdallah, Jr., F. Blasco, F. Dorchies, T. Caillaud, C. Bonté, C. Stenz, F. Salin, A. Y. Faenov, et al., Phys. Rev. A 66, 033204 (2002).

[19] J. Abdallah, Jr., G. C. Junkle-Vives, A. Y. Faenov, I. Y. Skobolev, T. A. Pikuz, A. I. Magunov, F. Blasco, C. Bonté, F. Dorchies, et al., J. Quant. Spectrosc. Radiat. Transf. 81, 3 (2003).

[20] J. Abdallah, Jr., G. Csanak, Y. Fukuda, Y. Akahane, M. Aoyama, N. Inoue, H. Ueda, K. Yamakawa, A. Y. Faenov, et al., Phys. Rev. A 68, 063201 (2003).

[21] J. P. Rozet, M. Cornille, S. Dobosz, J. Dubau, J. C. Gauthier, S. Jacquemot, E. Lamour, M. Lezius, D. Normand, M. Schmidt, et al., Phys. Scripta T92, 113 (2001).

[22] V. Bagnoud and F. Salin, Appl. Phys. B 70, S165 (2000).

[23] F. Dorchies, F. Blasco, T. Caillaud, J. Stevefelt, C. Stenz, A. S. Boldarev, and V. A. Gasilov, Phys. Rev. A 68, 023201 (2003).

[24] F. Blasco, C. Stenz, F. Salin, A. Y. Faenov, A. I. Magunov, T. A. Pikuz, and I. Y. Skobolev, Rev. Sc. Instrum. 72, 1956 (2001).

[25] G. Holzer, O. Wehrhan, J. Heinisch, E. Forster, T. A. Pikuz, A. Y. Faenov, V. M. Romanova, and T. A. Shelkovenko, Phys. Scripta 57, 301 (1998).

[26] V. Kumarappan, M. Krishnamurthy, D. Mathur, and L. C. Tribedi, Phys. Rev. A 63, 023203 (2001).

[27] M. V. Ammosov, N. B. Delone, and V. P. Krainov, Sov. Phys. JETP 64, 1191 (1986).

[28] T. Auguste, P. D'Oliveira, S. Hulin, P. Monot, J. Abdallah, Jr., A. Y. Faenov, I. Y. Skobolev, A. I. Magunov, 
and T. A. Pikuz, Sov. Phys. JETP Lett. 72, 38 (2000).

[29] K. J. Mendham, J. W. G. Tisch, M. B. Mason, N. Hay, and J. P. Marangos, Opt. Express 11, 1357 (2003).

[30] J. Zweiback, T. Ditmire, and M. D. Perry, Phys. Rev. A 59, R3166 (1999).

[31] F. Megi, M. Belkacem, M. Bouchene, E. Suraud, and G. Zwicknagel, J. Phys. B: At. Mol. Opt. Phys. 36, 273 (2003).

[32] W. Lotz, Z. Phys. 216, 241 (1968).

[33] I. Last and J. Jortner, Phys. Rev. A 62, 013201 (2000).
[34] M. S. Murillo and J. C. Weisheit, Physics Reports 302, 1 (1998).

[35] M. Smirnov and W. Becker, Phys. Rev. A 69, 013201 (2004).

[36] I. Last and J. Jortner, Phys. Rev. A 60, 2215 (1999).

[37] C. Rose-Petruck, K. J. Schafer, K. R. Wilson, and C. P. J. Barty, Phys. Rev. A 55, 1182 (1997).

[38] Database available on www.nist.gov 